\title{
MAGNETOGRAVIMETRIC SEPARATION IN A ROTATIONAL DEVICE
}

\author{
R.C. BUNGE ${ }^{\star}$ AND D.W. FUERSTENAU \\ Department of Materials Science and Mineral Engineering \\ University of California, Berkeley, CA 94720, USA
}

\begin{abstract}
Magnetogravimetric separation in a rotational device is a promising method for effecting sharp separation of minerals according to density. Separation is accomplished by two competing forces with opposite directions, namely the magnetic buoyancy and the centrifugal force. Magnetic buoyancy is experienced by particles which are suspended in a magnetic fluid when exposed to a non-homogeneous magnetic field. Since the magnetic buoyancy depends on particle volume whereas the centrifugal depends on particle mass, a purely gravimetric separation of non-magnetic particles can be accomplished. The process is able to achieve separation split-points

up to densities of $20 \mathrm{~g} / \mathrm{cm}^{3}$, but separation sharpness decreases with fine particle sizes. On a laboratory scale, magnetogravimetric separators can be used as an alternative to heavy liquid separation, and in industrial plants they may have promise as the final cleaning stage in gravity concentration circuits of precious metals.
\end{abstract}

(Received November 11, 1995, in final form February 2, 1996)

\section{INTRODUCTION}

Magnetogravimetric separation exploits the difference in the densities between commercially valuable minerals and the associated gangue. It is a true gravity concentration process. Within the domain of gravity concentration, heavy liquid separation is widely regarded as the "perfect" process with respect to separation sharpness. Unfortunately, heavy liquid separation is associated with serious practical problems, both economic and technological. Heavy liquids are expensive,

${ }^{\star}$ Present address: BMG Engineering AG, Ifangstrasse 11, 8952 Schlieren-Zurich, Switzerland 
often poisonous, viscous and, most of all, generally limited to split-point densities below $3 \mathrm{~g} / \mathrm{cm}^{3}$.

Magnetogravimetric separation may be a viable alternative. This paper introduces the basic theory of magnetogravimetric separation and demonstrates the capabilities of the process in general and those of a rotational device in particular.

\section{THE THEORY OF MAGNETIC BUOYANCY}

Magnetogravimetric separation is based on the balance of two competing forces acting in opposite directions, magnetic buoyancy and gravitation (or, as in the case of a rotational device, centrifugal force) [1 - 3]. Magnetic buoyancy depends on particle volume whereas gravitation is a function of particle mass (which depends on both volume and density). Accordingly, minerals can be separated with respect to their density. Magnetic buoyancy, which is not a phenomenon of common knowledge, is introduced in this section.

A non-magnetic particle suspended in a magnetic fluid which is exposed to a magnetic field, will experience a force repulsing it from the magnet, that is, magnetic buoyancy. This effect can be illustrated by an analogy with hydrostatic buoyancy.

Hydrostatic buoyancy (Fig. 1a) occurs in a fluid due to non-symmetric pressure distribution around a suspended particle. Pressure increases in the fluid container linearly with depth. While the forces generated on a suspended particle by lateral pressures cancel, the pressure difference below and above the particle will generate a net force opposing the direction of the gravitational force, hydrostatic buoyancy.

A similar effect is observed when a non-magnetic particle is suspended in a magnetic liquid (Fig. 1b) and introduced into a magnetic field. Obviously, hydrostatic buoyancy occurs here, too. However, we now consider only horizontal forces. The molecules of the magnetic fluid are attracted by the magnet which leads to a horizontal pressure gradient. Consequently, the lateral fluid pressure on the particles is non-symmetrical: it is repulsed from the magnet [4]. 

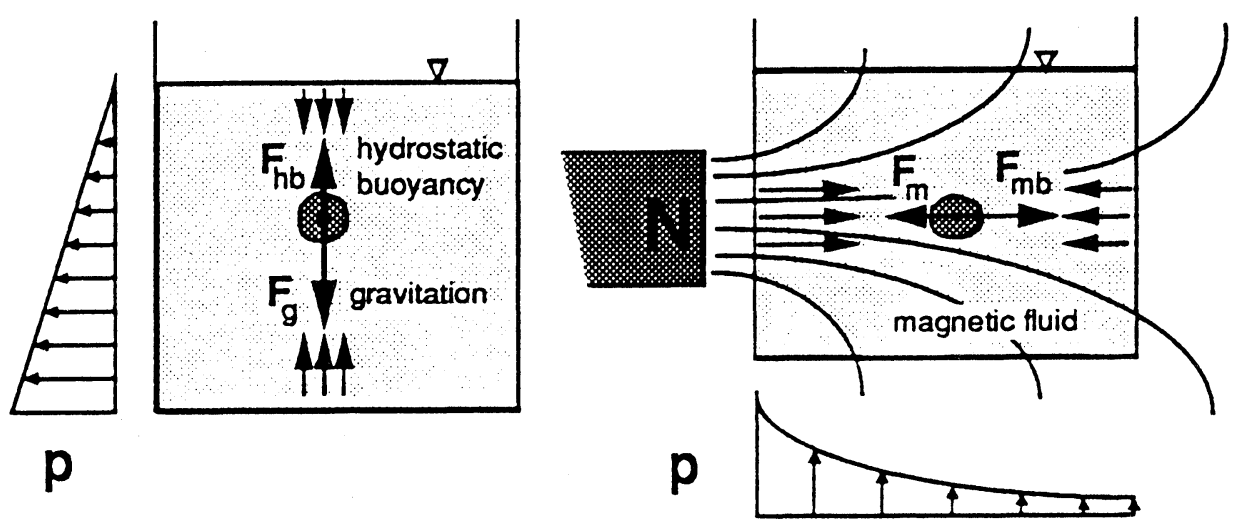

Fig. 1 (a) Hydrostatic buoyancy is due to a non-symmetrical hydrostatic pressure distribution around a suspended particle (b) Magnetic buoyancy due to a non-symmetrical "magnetic pressure" distribution around a suspended particle

The analogy between hydrostatic and magnetic buoyancy also extends to the mathematical description of both forces. A list of symbols is found at the end of this text.

$$
\begin{aligned}
& F_{h b}=V \rho_{\mathrm{f}} \mathrm{g} \\
& F_{\mathrm{mb}}=\mathrm{V} \chi_{\mathrm{f}} \mu_{\mathrm{o}} \mathrm{H} \operatorname{gradH}
\end{aligned}
$$

Equation (1a) describes hydrostatic buoyancy while eq. (1b) describes the magnetic buoyancy. Both forces depend on three terms: the particle volume $V$ (which is also the volume of the replaced fluid), a property of the fluid (density $\rho_{\mathrm{f}}$ or magnetic susceptibility $\chi_{\mathrm{f}}$ ), and the third term describing a field specific to the selected fluid property (gravitational field $g$, magnetic field $\mu_{0} \mathrm{H}$ gradH).

If the buoyancy forces (hydrostatic, magnetic) oppose the attractive forces (gravitation, magnetic), gravity concentration of essentially non-magnetic 
minerals can be accomplished on a device such as the one sketched in Fig. 2a [5]. The vertical force balance in this case is expressed by:

$$
F_{y}=F_{m b}+F_{h b}-F_{g}=V\left[\chi_{f} \mu_{0} H \operatorname{gradH}+g\left(\rho_{f}-\rho_{p}\right)\right]
$$
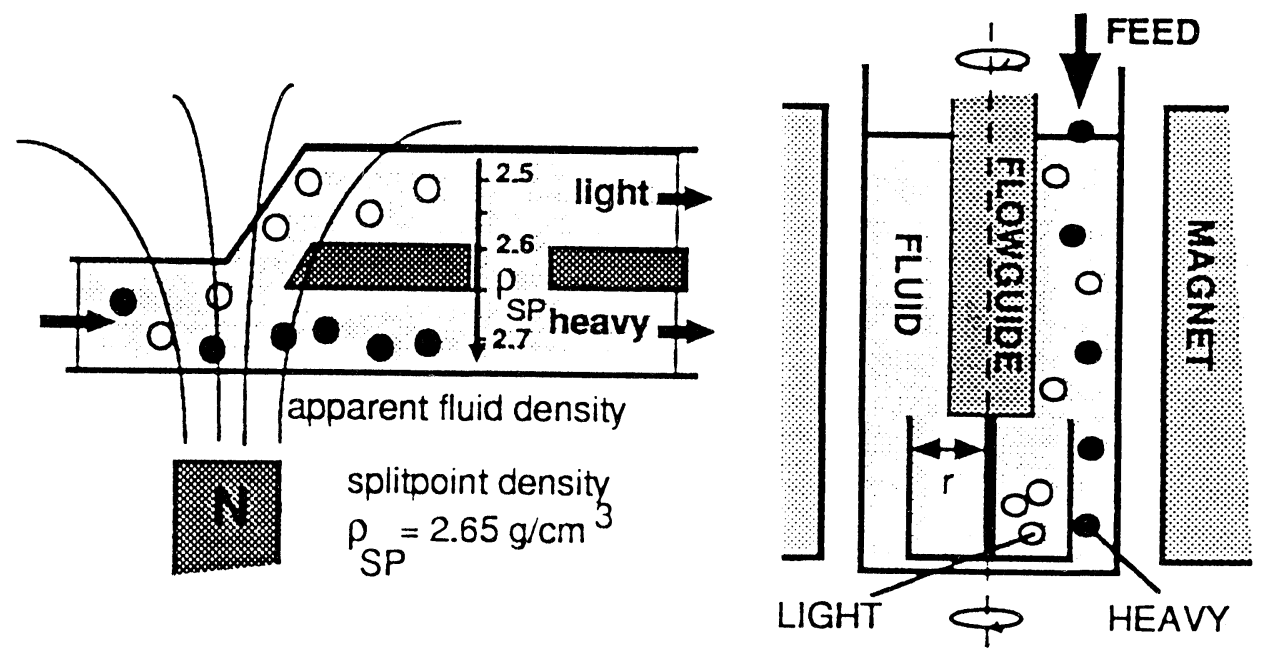

Fig. 2 (a) Both magnetic buoyancy and gravitation determine the equilibrium distance of a non-magnetic particle from the magnet resulting in magnetogravimetric separation (b) Introduction of a rotational device to provide centrifugal force instead of gravitation

Because in ferrofluids the saturation magnetisation is reached at the field strengths typical for magnetogravimetric separators, the term $\chi_{\mathrm{f}} H$ can be replaced by $M_{\mathrm{f}}$, the fluid magnetisation. Equilibrium of vertical forces $\left(F_{\mathrm{y}}=0\right)$ yields:

$$
\rho_{\mathrm{sp}}=\frac{\mathbf{M}_{\mathrm{f}} \mu_{\mathrm{o}} \mathrm{gradH}}{\mathrm{g}}+\rho_{\mathrm{f}}
$$

The density of the equilibrium is termed split-point density. Particles with densities higher than this split-point sink, while particles with lower densities float. 


\section{THE MAGSTREAM SEPARATION PROCESS}

The research presented here was carried out in a laboratory-size batch model of a commercial rotational magnetogravimetric separator Magstream 100.

\section{The Fluid}

Although in principle the separator could work with any magnetic fluid, the manufacturer recommends that a particular fluid be used. This fluid is an aqueous suspension of colloidal magnetite particles which is stable over months. However, since the fluid is biodegradable at $\mathrm{pH}$ below 10 , high $\mathrm{pH}$ must be maintained to avoid bacterial degradation.

The fluid is delivered as a concentrate of density of $1.2 \mathrm{~g} / \mathrm{cm}^{3}$ and the desired magnetisation is then adjusted by dilution with water. The fluid magnetisation is proportional to the fluid density since the amount of colloidal magnetite affects both magnetisation and fluid density. The manufacturer supplies a magnetisation chart, that is a plot of magnetisation versus fluid density, with every batch of fluid.

Because the relative amounts of magnetite and stabilisers slightly differ for different batches of fluid, the slopes of the regression lines on these charts can also vary. By using the calibration charts, an operator has a very simple means of determining the fluid magnetisation which is difficult to measure directly. Instead, fluid density is measured with a hygrometer and the corresponding magnetisation value is extracted from the chart.

\section{The Separator}

The rotational separator operates in principle as already outlines in Fig. 2a. However, the Magstream device rotates around a vertical axis and hence the gravitation is replaced by the centrifugal force (Fig. $2 b$ ) $[6,7]$.

The Magstream 100 is furnished with an annular permanent magnet. A particular feature of this device is a magnetic field gradient that decreases linearly with distance from the magnet according to: 
where $r$ is the septum radius. The manufacturer specifies $k$ as $1324 / \mu_{0}\left[\mathrm{~A} / \mathrm{m}^{2}\right]$.

In operation, the separator duct (containing the flow guide and the attached inner cup) rotates at an adjustable speed. The particles experience the centrifugal force that drives them from the centre toward the magnet and magnetic buoyancy which is directed inward. Neglecting the drag forces (static approach) yields the following equations:

$$
\begin{aligned}
& \mathrm{F}_{\mathrm{mb}}=\mathrm{VrMk} \\
& \mathrm{F}_{\mathrm{c}}=\mathrm{V}\left(\rho_{\mathrm{p}}-\rho_{\mathrm{f}}\right) \omega^{2} \mathrm{r}
\end{aligned}
$$

In analogy with Eqs. (2) and (3), it follows that

$$
\mathbf{F}_{\mathbf{x}}=\mathbf{F}_{\mathrm{mb}}-\mathbf{F}_{\mathbf{c}}=\operatorname{Vr}\left[\mathbf{M k}-\left(\rho_{\mathrm{p}}-\rho_{\mathrm{f}}\right) 4 \pi^{2} \mathrm{n}^{2}\right]
$$

and for the equilibrium

$$
\mathrm{F}_{\mathrm{mb}}=\mathrm{F}_{\mathrm{c}} \rightarrow \mathrm{F}_{\mathrm{x}}=0 \rightarrow \rho_{\mathrm{sp}}=\rho_{\mathrm{f}}+\frac{\mathrm{kM}}{4 \pi^{2} \mathrm{n}^{2}}
$$

The split-point density is again the density calculated for the equilibrium situation. A particle species of exactly this density reports in equal amounts to both products (inner and outer). The rotational speed corresponding to the split-point is

$$
\mathrm{N}=\mathrm{K}\left[\frac{\mathrm{M}}{\rho_{\mathrm{sp}}-\rho_{\mathrm{f}}}\right]^{\frac{1}{2}}
$$

where all constants are collected in $K$ whose numerical value is 347 .

To separate two minerals of different density, the split-point is chosen at a density between those of the minerals. This is accomplished by selecting the rotational speed according to eq. (9). The light mineral, due to buoyancy overriding the centrifugal force, will be driven toward the center and report to the inner cup. In 
contrast, the centrifugal force dominates buoyancy on heavy particles driving them toward the outer wall. The heavy particles finally collect at the bottom of the separator duct. Since, as discussed above, magnetisation is a linear function of fluid density, the magnetisation $M$ is substituted by

$$
\mathbf{M}=\mathbf{a}^{\prime}\left(\rho_{\mathrm{f}}-\rho_{\mathrm{w}}\right)
$$

where $a^{\prime}$ is the slope of the regression line on the calibration chart. Assuming the density of water as $1 \mathrm{~g} / \mathrm{cm}^{3}$, we derive for the split-point and the corresponding rotational speed

$$
\begin{aligned}
& \rho_{\mathrm{sp}}=\rho_{\mathrm{f}}+\left(\rho_{\mathrm{f}}-1\right) \frac{\mathrm{K}^{2}}{\mathrm{~N}^{2}} \\
& \mathrm{~N}=\mathrm{K}\left[\mathrm{a}, \frac{\rho_{\mathrm{f}}-1}{\rho_{\mathrm{sp}}-\rho_{\mathrm{f}}}\right]^{\frac{1}{2}}
\end{aligned}
$$

With this kind of magnetogravimetric separator, ideally particle size should not affect the separation split-point, which is in contrast to most gravity concentration devices where inherent difficulties occur when separating coarse light particles from small heavy ones, that is, particles of equal terminal settling velocity.

All previous considerations are based on the assumption of essentially non-magnetic particles. If, however, the particles are magnetic, some attraction toward the magnet is superimposed with the discussed forces. In this case separation can be affected in two ways. If the magnetic particles happen to be the desired heavy mineral, the separation would be more effective due to the additional outward force. If, in contrast, the magnetic particles are the light fraction, separation efficiency would suffer. The light magnetic particles would, instead of collecting in the inner cup, move toward the magnet (outer wall) and consequently report to the outer product where they contaminate the heavy non-magnetics.

Also, strongly magnetic particles are forcefully attracted by the magnet and tent to stick to the outer wall instead of sliding downward into the concentrate chamber (an effect the manufacturer termed "pinning"), thus eventually clogging 
the separator. Therefore, ferromagnetic minerals should be removed from the feed prior to separation.

The simplest case is the separation of two fully liberated minerals with known densities, Here, the first step is the determination of a suitable split-point. If a single pass separation is desired the split-point should be chosen just in the middle between the densities of the two feed minerals. After selecting an adequate fluid density (for reasons discussed later this is usually approximately $1.07 \mathrm{~g} / \mathrm{cm}^{3}$ ) the required rotational speed is calculated from eq. (12).

After each run the fluid can be recycled by filtering the products. Inevitably some fluid will be lost as the residual moisture of the filtered products. Depending on mineral particle size, this loss can run as high as $250 \mathrm{ml}$ per $\mathrm{kg}$ of the processed mineral.

\section{CHARACTERISATION OF SEPARATOR PERFORMANCE}

A separation is usually characterised by a "mean" value and some value representing the scatter. Both values can be determined from performance curves. A performance curve is a plot of the recovery of a single mineral feed versus the corresponding separator setting, in our case the split-point density (which depends on the selected rotational speed). For determination of performance curves, the feed is assumed "ideal" (single-size non-magnetic mineral, fully liberated, spherical particles) and all imperfection is attributed to the separator itself.

Some parameters of possible influence on the process are those listed below:

Particle characteristics:

density, size, degree of liberation, shape, magnetic susceptibility, surface properties (wettability)

Fluid characteristics: magnetisation, density, viscosity, stability

Separator characteristics: geometry of installation, magnetic field (strength and gradient) 
Process characteristics:

feedrate, rotational speed.

The identification of the influence the italicised parameters have on the separator performance is the subject of the following considerations. All other parameters were assumed to be either constant or to have negligible influence on the separator performance.

To link the parameter under test unambiguously to the effect on separator performance, we varied, where possible, only one parameter at a time and observed its effect on the performance curve.

It has already been pointed out that some parameters, for example fluid magnetisation and fluid density are not independent. Since the fluid magnetisation is a function of fluid density, the effect of a variation in fluid density on separator performance is not necessarily primarily due to a change in magnetisation but could also be due to a shift in density. However, a minute variation in fluid density apparently affects the magnetisation considerably.

The increase in hydrostatic buoyaricy due to increased fluid density can therefore be neglected in comparison with increased magnetic buoyancy generated by magnetisation. Similar considerations hold for fluid viscosity which is also linked to fluid density and therefore to magnetisation.

\section{EXPERIMENTAL RESULTS}

In this section we present a number of figures to illustrate how some selected input parameters affect the separator performance curves. Figure 3 shows performance curves for two sizes of quartz. As already discussed, according to the foregoing equations, the separation should not depend on particle size (volume cancels in the derivation of the split-point density).

Indeed, the "mean split-point densities" (at 50\% recovery to either fraction) are approximately equal for both size fractions. Moreover, the mean split-point densities derived for each mineral from the plots are basically in accordance with 


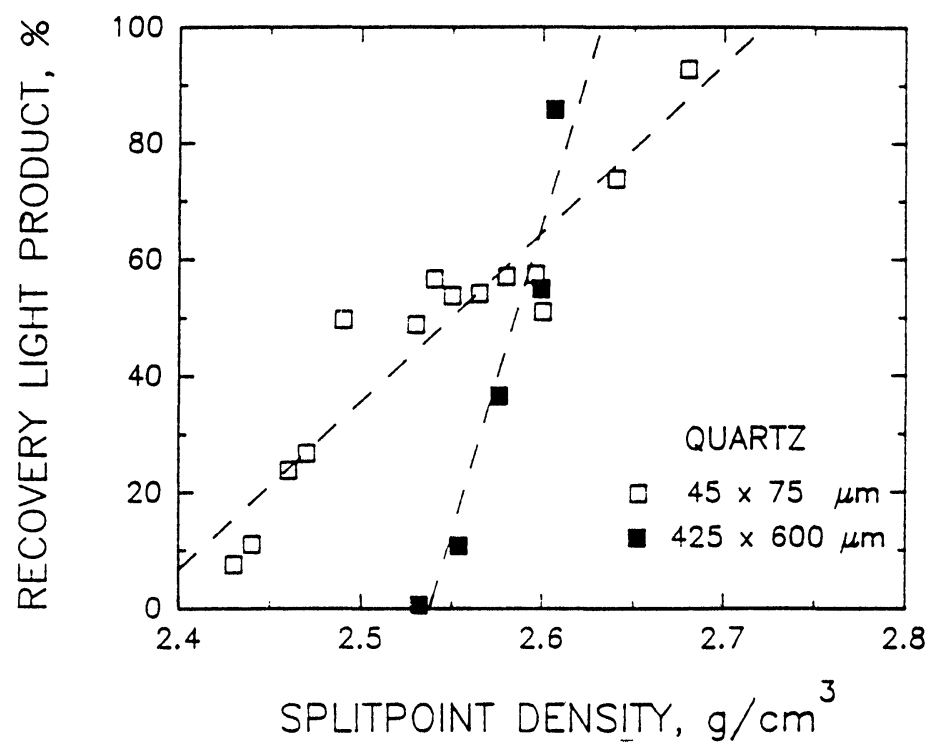

Fig. 3 Separator performance curves for quartz at two particle sizes: the separation sharpness increases with particle size.

the actual mineral densities. Ideally, since single minerals are processed, the feed should report with equal amounts to each product fraction if the split-point density equals the mineral's density, which means that the horizontal forces (buoyancies and centrifugal force) on the particles just cancel.

Actually, we observe a mean split-point density that deviates from the known particle density. The performance curves are slightly shifted towards higher recoveries. For example, in Fig. 3 we observe for the fine size fraction that at true density of quartz $\left(2.65 \mathrm{~g} / \mathrm{cm}^{3}\right)$ the recovery of $80 \%$ instead of the expected $50 \%$ is obtained. This offset varies with various batches of magnetic fluid and seems also to depend on the size of the particles processed. The reason for this offset is not quite clear.

In contrast to the mean split-point, the separation sharpness as represented by the slope of the performance curve is affected by particle size. It increases with 
increasing particle size. An explanation for this behavior is not directly obvious from the basic process theory presented here. In the context of this paper, it may suffice to assume that the long residence time of the fines will subject them to stochastic events which tend to disperse the settling particles over the separation chamber, hence worsen the separation. A detailed account of this effect is discussed elsewhere [5].

In Fig. 4 the effect of varying feed rate on the separation sharpness is shown for fine barite. Over a wide range of feed rates, the separation sharpness is largely unaffected. The effect is even less pronounced for coarser particles. Since the Magstream 100 is a laboratory device, high feed rates are not a major objective. Accordingly, typical feed rates lie in the order of 0.1 to $0.2 \mathrm{~g} / \mathrm{s}$. The increase in separation efficiency with feed rates lower than $0.1 \mathrm{~g} / \mathrm{s}$ is not very pronounced, that is, much less than the effect of other parameters such as particle size.

According to Fig. 5, the fluid magnetisation has some impact on the separation sharpness. As discussed above, the fluid magnetisation is a function of the fluid density, the parameter actually measured.

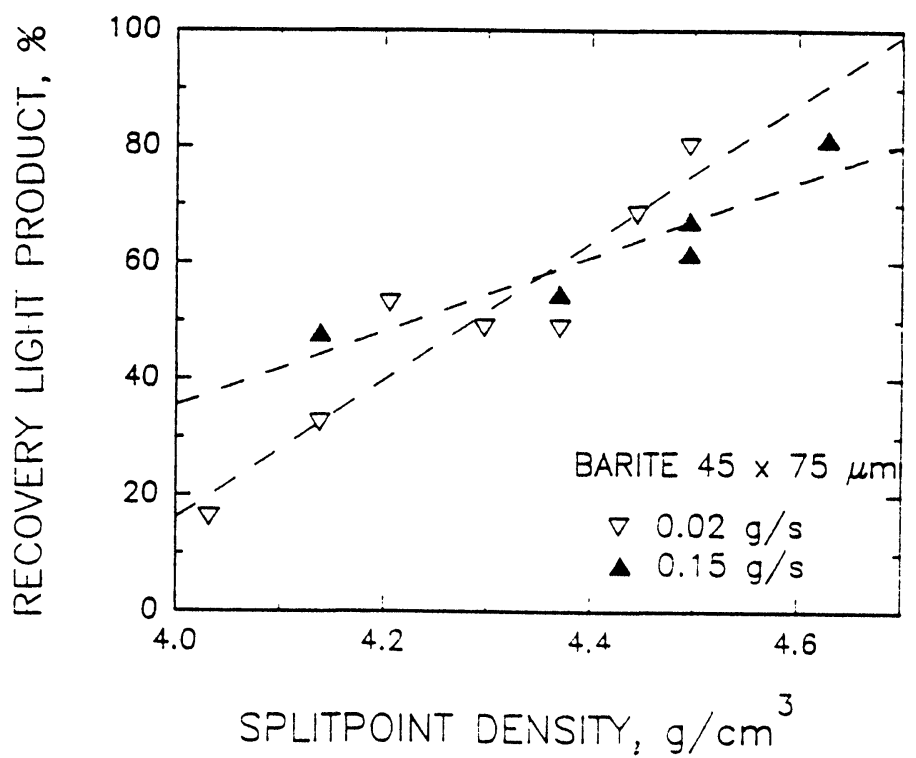

Fig. 4 Separator performance curves for barite at two feed rates: increasing feed rate adversely affects separation sharpness 
It has also been discussed that a change in fluid density alone would not be expected to influence the separation appreciably. We can therefore assume that the increase in the separation sharpness with more concentrated fluids is actually due to increased fluid magnetisation. In this case all deterministic forces involved are higher and the separation sharpness increases due to a relative suppression of stochastic events.

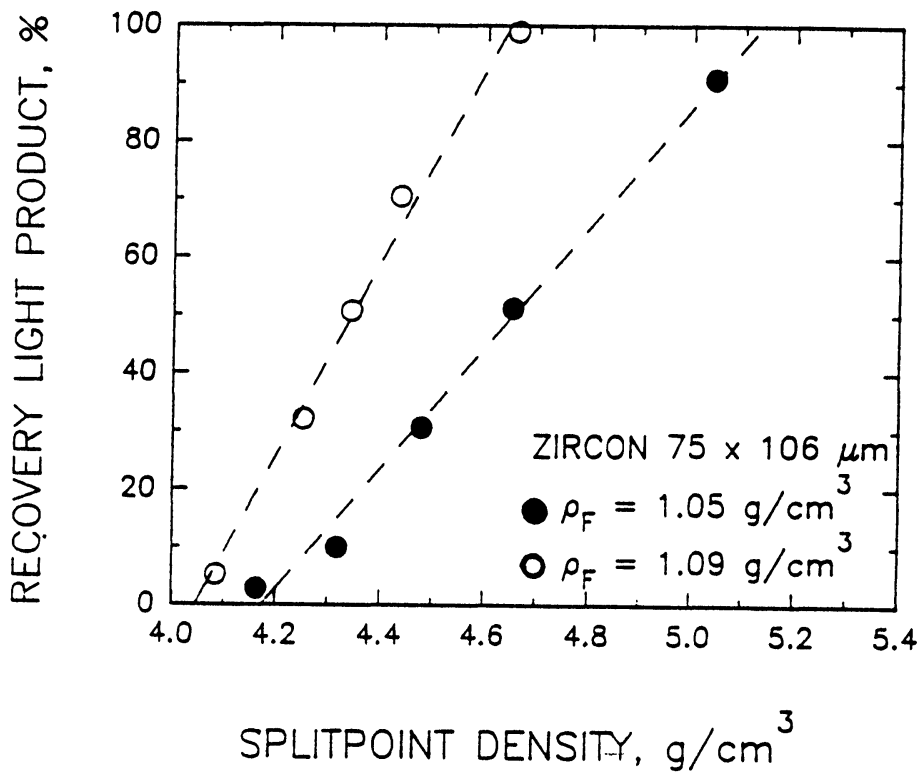

Fig. 5 Separation performance curves for zircon at two fluid densities (magnetisations): separation sharpness increases with fluid magnetisation

Although higher fluid densities, that is higher fluid magnetisations, bring about better separation sharpness, in practice the exploitation of this effect is limited by the increasing fluid viscosity. At fluid densities exceeding $1.0 \mathrm{~g} / \mathrm{cm}^{3}$ the viscosity increases very sharply, therefore the settling velocity of fine particles becomes too slow for reasonable throughputs. By and large, we have observed that a fluid density of $1.07 \mathrm{~g} / \mathrm{cm}^{3}$ offers a good compromise between throughput capacity and the separation sharpness for most applications. 
Summarising, we conclude that:

(i) the measured mean separation split-points correspond sufficiently well with the tabulated mineral densities and

(ii) the separation sharpness of the Magstream 100 is mainly controlled by particle size and fluid magnetisation.

The performance curves presented in Figures 3, 4 and 5 were obtained with single mineral feeds by varying the rotational speed, that is, in effect by varying the calculated split-point density. We also carried out actual separations of feed mixtures Figure 6 shows the separation results for processing a fluorite/limestone mixture. Both minerals were size-distributed between 75 and $600 \mu \mathrm{m}$. The

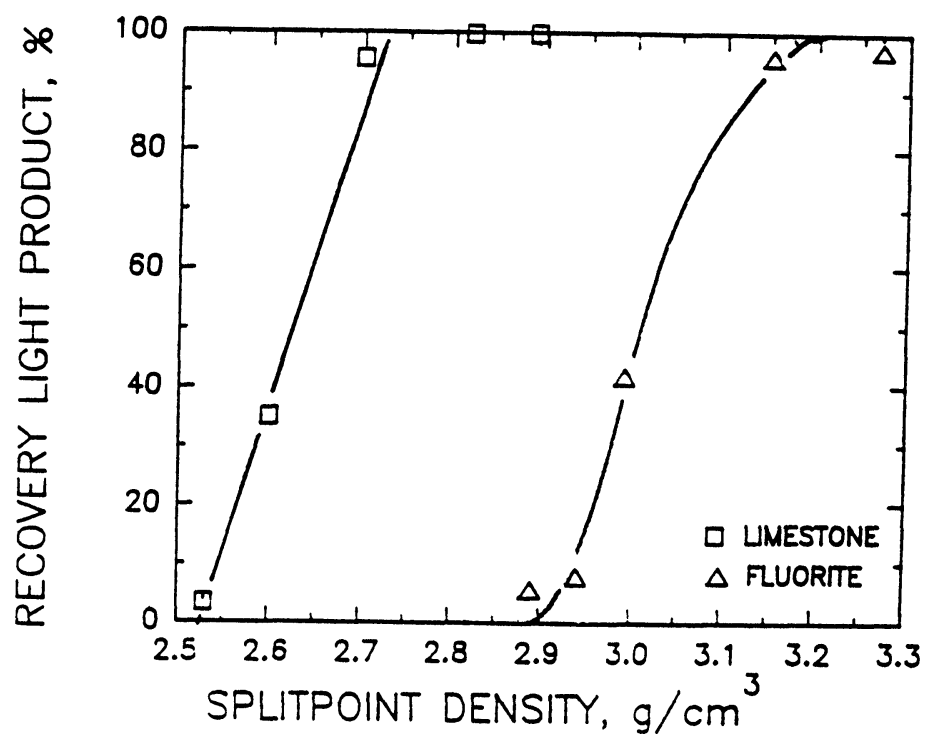

Fig 6 Separation of a fluorite/limestone mixture: complete separation is achieved at a calculated split-point of $2.8 \mathrm{~g} / \mathrm{cm}^{3}$.

optimum separation result was achieved at a separator setting of $2.8 \mathrm{~g} / \mathrm{cm}^{3}$, that is, a rotational speed corresponding to the split-point density according to eq. (11). 


\section{PREDICTED SEPARATOR PERFORMANCE CURVES}

We have developed a model for the Magstream 100 device to predict the outcome of separations at given operating and feed parameters. This paper does not discuss the modelling process (for details see [5]) but presents some predictions for the performance curves of single minerals.

Assuming that the minerals to be separated do not mutually interfere in the separation chamber, a valid assumption in view of the very dilute system, the outcome of actual separations can be inferred from the predicted individual performance curves. The percentage recovery $R$ is linked with the calculated split-point density $\rho_{\text {sp }}$ as shown in eq. (13) (all densities in $\mathrm{g} / \mathrm{cm}^{3}$, particle size $d$ in $\mu \mathrm{m}, a^{\prime}$ is usually approximately 26$)$ :

$$
\mathrm{R}=7.8 \mathrm{da},\left[\frac{\left(\rho_{\mathrm{f}}-1\right)^{2}}{\left(\rho_{\mathrm{p}}-\rho_{\mathrm{f}}\right) 10^{10}\left(\rho_{\mathrm{f}}-1\right)}\right]^{\frac{1}{2}}\left[1 \frac{\rho_{\mathrm{p}}-\rho_{\mathrm{f}}}{\rho_{\mathrm{sp}}-\rho_{\mathrm{f}}}\right]+50
$$

The split-point density can be expressed as a function of rotational speed according to eq. (11).

In Figures $7 \mathrm{a}, \mathrm{b}$ and $\mathrm{c}$ some predictions (after eq. (13)) are plotted which may help the user to determine easily the operating variables to be chosen for a desired separation efficiency. The use of these graphs is illustrated by the following example.

Consider separation of quartz $(\rho=2.65)$ from fluorite $(\rho=3.2)$, both of discrete particle size $100 \mu \mathrm{m}$. The fluid density is 1.05 (see Fig. 8 which is an enhanced section of Fig. 7b). Interpolating between the lines plotted on this graph we obtain the dashed lines for quartz and fluorite. By selecting a rotational speed corresponding to a split-point of $2.9 \mathrm{~g} / \mathrm{cm}^{3}$, a reasonably good separation can be expected (let for instance $a^{\prime}=26$, then $N$ should be equal to $291 \mathrm{rpm}$, according to eq. (12)).

As we have pointed out above, the performance curves are slightly offset towards higher recoveries; the recovery at $\rho_{\mathrm{p}}=\rho_{\mathrm{sp}}$ is above $50 \%$. This offset is considered by the following empirical correction in our model: 


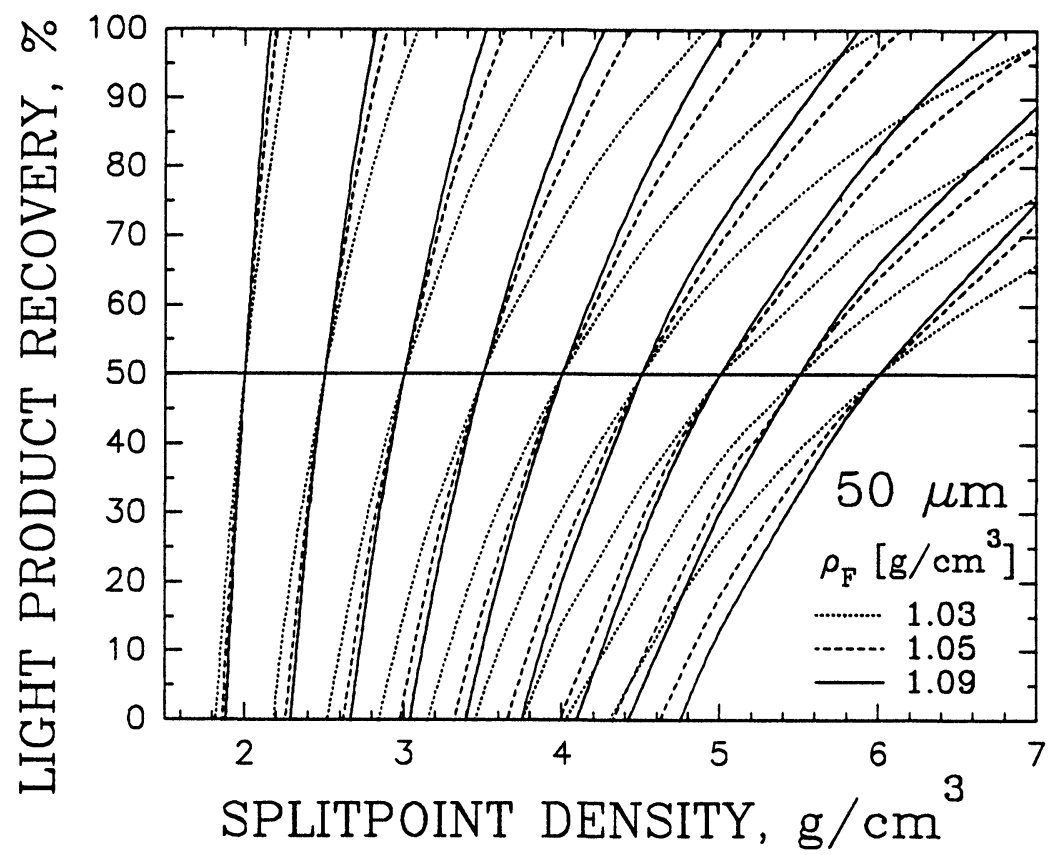

Fig. 7a Predicted separator performance curves for $50 \mu \mathrm{m}$ particles

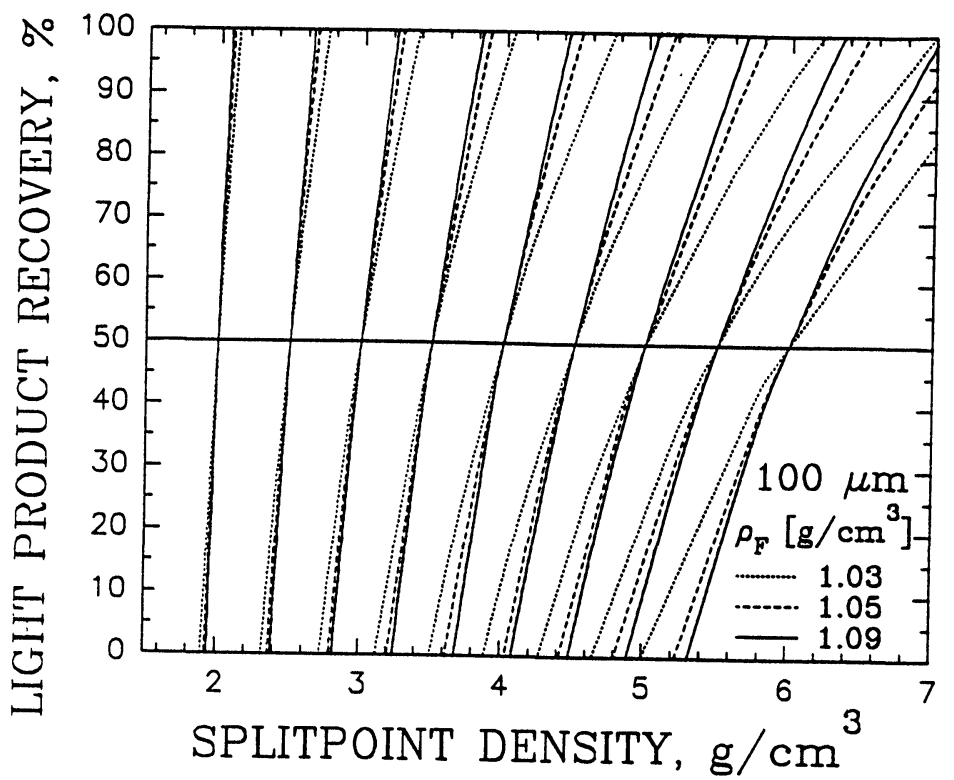

Fig. 7b Predicted separator performance curves for $100 \mu \mathrm{m}$ particles 


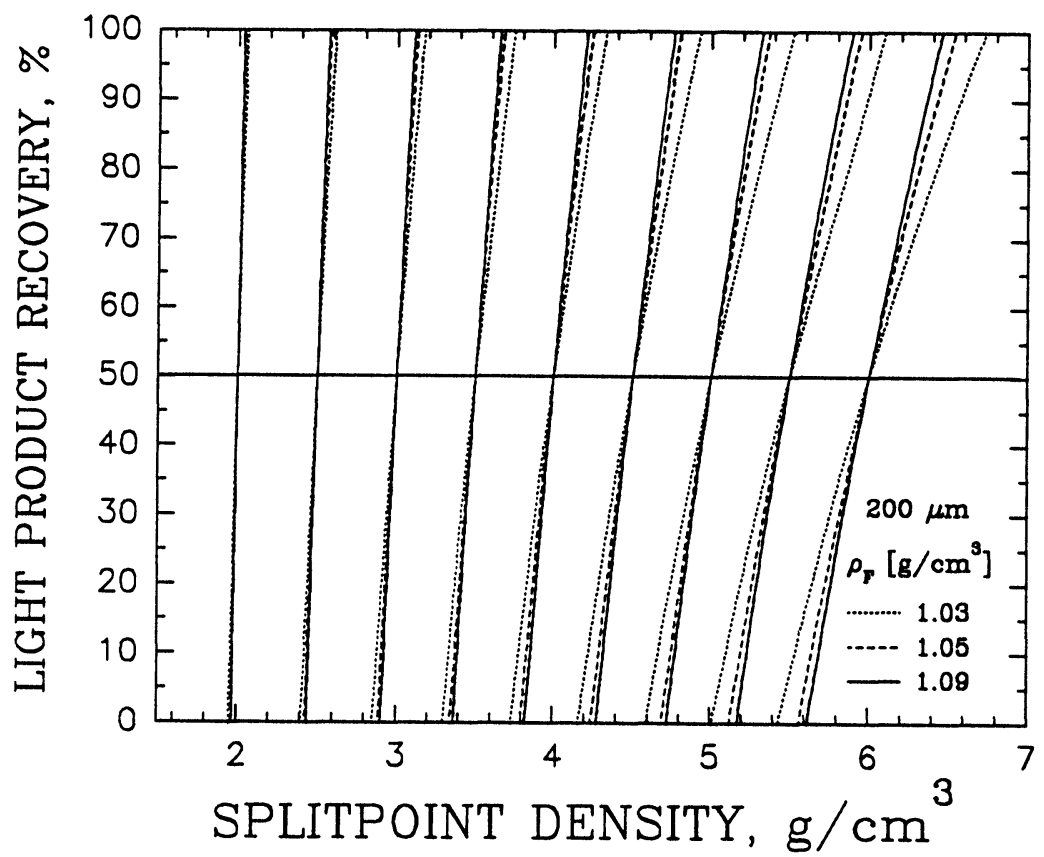

Fig. 7c Predicted separator performance curves for $200 \mu \mathrm{m}$ particles

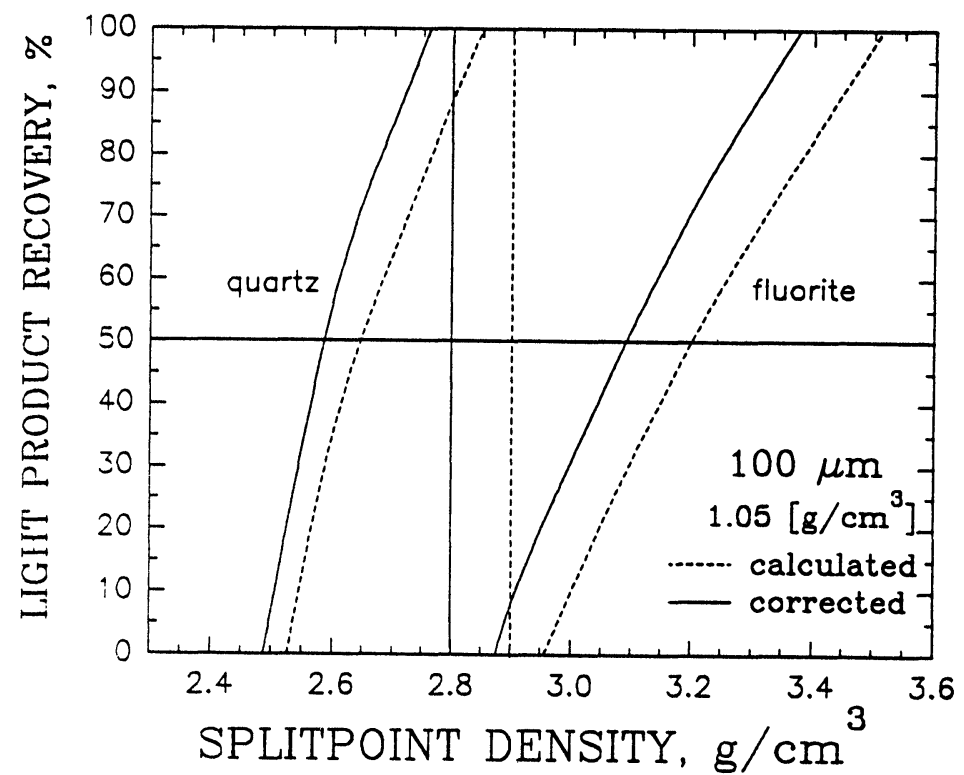

Fig. 8 Predictions of separator performance curves for $100 \mu \mathrm{m}$ particles at fluid density $1.05 \mathrm{~g} / \mathrm{cm}^{3}$. The corrected curves (heavy lines) are offset to higher recoveries 


$$
\mathrm{R}_{\text {corr }}=\mathrm{R}-50+5.6 \sqrt{\mathrm{d}}+4.9 \times 10^{10}\left(\rho_{\mathrm{f}}-1\right)
$$

The offset appears to depend mainly on particle size $d$ and the fluid viscosity which is represented by the exponential term. The latter may depend partly on the batch of the fluid used. Also, the separator geometry may affect the offset.

We recommend that for each batch of fluid a calibration curve be produced by processing a few samples of material with known particle size and density. From this curve the constants in eq. (14) can be reevaluated. For example, using eq. (14), the correction for the above example $\left(100 \mu \mathrm{m}, 1.05 \mathrm{~g} / \mathrm{cm}^{3}\right)$ is calculated to be $21 \%$, represented in Fig. 8 by solid lines. Consequently, the split-point shifts to 2.8 which corresponds to the rotational speed of $300 \mathrm{rpm}$.

In contrast to the above example, minerals will generally be size-distributed. In that case one would take the results of a particle size analysis and plot the performance curves for each size fraction individually. After normalising the curves with respect to the relative amount of material present in the respective size fraction, the performance curves for each size fraction are added individually for each mineral. The resultant performance curve for a size-distributed feed will have a shape of a polygon (since the performance curves for single particle sizes are almost straight lines).

Note that the "tails" of the resultant performance curves consist of mainly fine material. In Fig. 9 the resultant performance curve is shown for a mineral consisting of two discrete sizes, each making up $50 \%$ of the feed. The split-point density at the $50 \%$ recovery (that is, the split-point density of the material) is equal for both particle sizes. However, the finer size produces more scatter (as indicated by a performance curve with reduced slope). By normalising the performance curves for each fraction with $50 \%$ and adding both curves, the polygonal resultant performance curve is obtained.

If the tails of the performance curves for two size-distributed minerals overlap, the separation in the Magstream 100 will be incomplete (the shaded area in Fig. 10). As we have shown, these tails consist of predominantly fine material. While the coarse fractions of each mineral are separated reasonably well, it is the fine sizes that are recovered together, regardless of the split-point density chosen. 


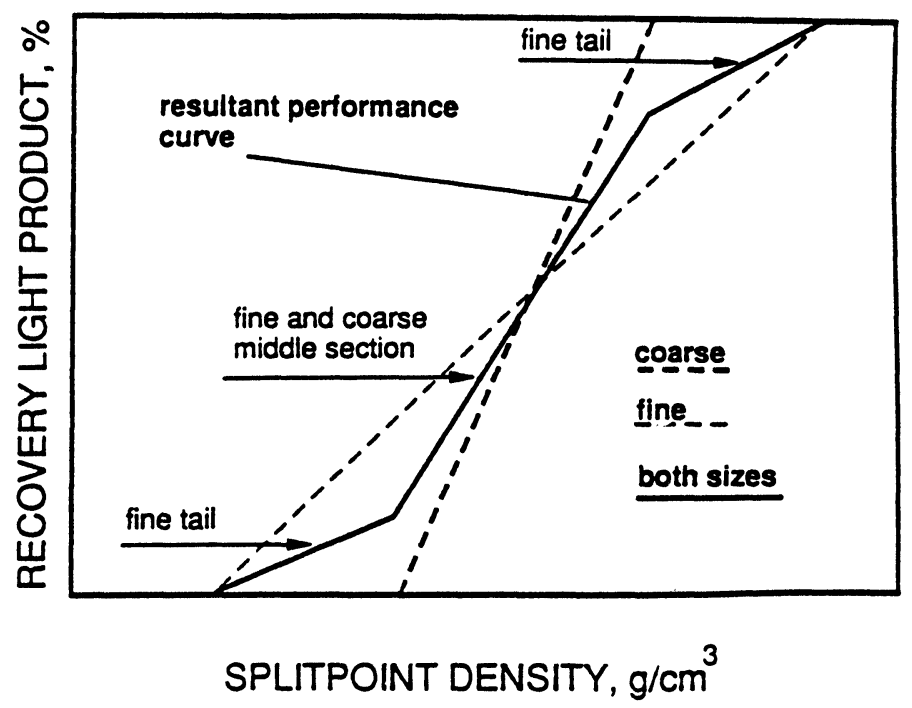

Fig. 9

The resultant performance curve is obtained by normalising and adding individual performance curves of two discrete particle size fractions. The tails of the resultant curve consist of fine materials.

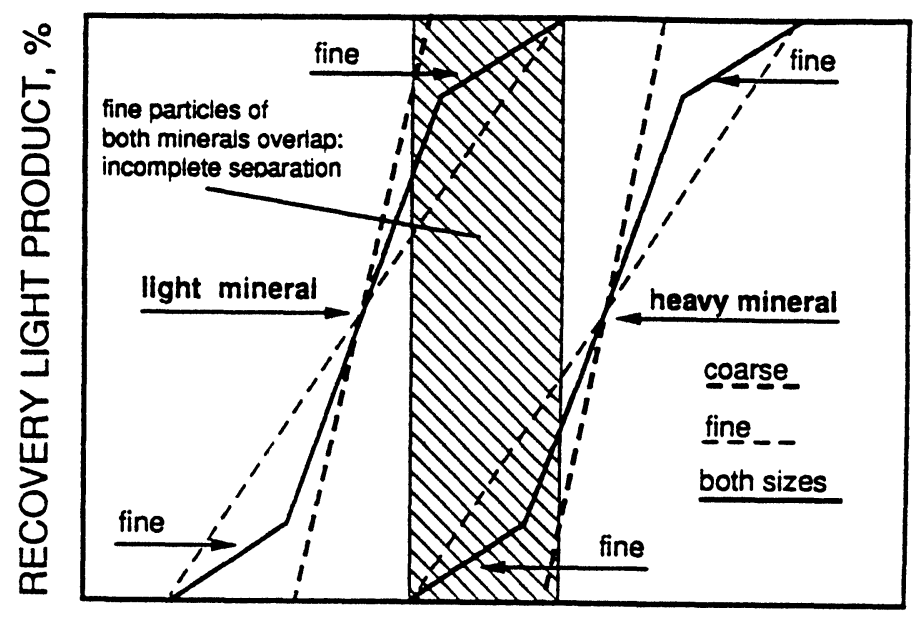

\section{SPLITPOINT DENSITY, $\mathrm{g} / \mathrm{cm}^{3}$}

Fig. 10 Incomplete separation of two minerals: the tails of both resultant performance curves overlap. 
Therefore, the amount of fine material in a size-distributed feed determines the overall sharpness of separation. Considering the fact that different size fractions may have different offsets, it may be advisable, if a maximum separation sharpness is crucial, to classify the feed material and process the size fractions each at its optimum separator setting.

While particle sizes above $200 \mu \mathrm{m}$ can be separated very sharply, the separation efficiency drops off rapidly for particles smaller than $50 \mu \mathrm{m}$. The above considerations apply primarily to separations in a single run but can be expanded to processing the feed in multiple runs. In this manner the separation sharpness can be enhanced considerably.

Let us consider the separation of $50 \mu \mathrm{m}$ material comprising two minerals (each $50 \%$ by mass), one of density 4.0 and the other of density 5.0 . Figure 7 a shows that even at the highest feasible fluid density (1.09) the curves overlap. Consequently, no separator setting can be found which would allow for a complete separation in one run.

Now let us assume that the feed is processed in a fluid of density 1.05 at a split-point setting of 5.2. According to Fig. 7a, 100\% of the light material and approximately $55 \%$ of the heavy mineral would report to the light product bin. The remaining $45 \%$ of the heavy mineral is recovered in the heavy product which is not contaminated by any light material. Consequently, by reprocessing the light material from the first run, the second run yield another $45 \%$ of the heavy mineral in the heavy product bin.

Thus, by successively depleting the mineral mixture of the heavy mineral fraction, only five runs would be required to produce an uncontaminated heavy product and a light product with only approximately $5 \%$ of the heavy mineral contamination.

\section{POSSIBLE APPLICATIONS AND FUTURE ASPECTS}

The Magstream process appears to be feasible at both laboratory and industrial scales. A possibility of achieving sharp gravity separations at fine particle sizes is 
certainly a promising feature. Nevertheless, as for any process, magnetogravimetric separation has disadvantages which limit its applicability. First, inevitably some fluid will be lost during operation through its adherence to the products of separation. This loss can be limited by washing the tailings and reconcentrating the resulting diluted fluid in a high-gradient magnetic separator.

While the loss of fluid is primarily an economic disadvantage, major technological problem lies in low throughput capacity of magnetogravimetric separators. Even if superconductors were used, the separation chamber would be comparatively small. Also, highly concentrated fluids (which would be required for sharp separation of fine feeds) are also very viscous, a possible problem when separating the fines.

The application on laboratory scale would not be appreciably affected by the cost of the fluid or the limited throughput. In contrast to most other gravity separation processes, magnetogravimetric separation has a great advantage of a defined link between the operating parameters and the separator performance (rotational speed and split-point). This guarantees good reproducibility (which is, for instance, not the case, for shaking tables).

In this respect only heavy liquid separation is comparable which is, however, practically limited to separation densities below $3 \mathrm{~g} / \mathrm{cm}^{3}$. In the case of high split-point densities, as required for mineralogical analysis of heavy minerals and for liberation studies of heavy minerals and for liberation studies, magnetogravimetric separation is the only process capable of achieving a high degree of separation sharpness.

On industrial scale, the sharpness may not be as crucial as the throughput capacity. Due to these limitations, only low throughputs of a high value material make the separation economically feasible. Ideal materials are those that occur fully liberated in placers, such a gold, platinum and diamonds, as well as tin and tungsten minerals. These could be pre-concentrated on traditional devices such as spirals or cones, and then further processed with a magnetogravimetric separator.

\section{ACKNOWLEDGMENTS}

The authors wish to gratefully acknowledge the full support from the US Bureau of Mines for Grant 1194106 to the California Mining and Mineral Resources Research 
Institute. We would also like to extend out thanks to the Intermagnetics General Corporation for providing the Magstream 100 used in this work.

\section{REFERENCES}

[1] U.T. Andres: Magnetohydrodynamic and Magnetohydrostatic Methods of Mineral Separation. Keter Publishing House, Jerusalem 1976

[2] G. Hartfeld: Magnetohydrostatische Sortierung im Feinkornbereich. Dissertation TU Clausthall, 1986

[3] H. Schubert: Aufbereitung fester mineralischer Rohstoffe, vol. 2, VEB Leipzig 1977

[4] R.C. Bunge and D.W. Fuerstenau: Mineral separations with a magnetogravimetric separator. Preprint AIME/SME Ann. Meeting, Salt Lake City, UT, February 1990

[5] R.C. Bunge and D.W. Fuerstenau: Modeling cross-flow separations. Int. J. Min. Proc. (submitted for publication 1993)

[6] M.S. Walker and A.L. Devernoe: Mineral separations using rotating magnetic fields. Int. J. Min. Proc, 31 (1991), 195-216

[7] M.S. Walker et al.: "Mainly gravity" separations using magnetic fluids under rotation. Proc. XVII IMPC, Vol. III, Dresden, Germany, September 1991

\section{LIST OF SYMBOLS}

a' slope of regression line in magnetisation chart

$\mathrm{F}_{\mathrm{hb}} \quad$ hydrostatic buoyancy

$\mathrm{F}_{\mathrm{mb}} \quad$ magnetic buoyancy

$\mathrm{F}_{\mathrm{c}} \quad$ centrifugal force

$\mathrm{F}_{\mathbf{x}} \quad$ resultant horizontal force

$\mathrm{F}_{\mathrm{y}} \quad$ resultant vertical force

g gravitational constant

$\mathrm{H}$ magnetic field strength

k constant

K constant

$\mathrm{M}_{\mathrm{f}} \quad$ fluid magnetisation

n revolutions/s

$\mathrm{N} \quad$ revolutions/min

$\mathrm{R}$ recovery in $\%$

$\mathrm{R}_{\text {corr }} \quad$ offset-corrected recovery

$r$ septum radius

V particle volume

$\chi_{\mathrm{f}} \quad$ magnetic susceptibility 


$\begin{array}{ll}\mu_{0} & \text { magnetic permeability } \\ \rho_{\mathrm{f}} & \text { fluid density } \\ \rho_{\mathrm{p}} & \text { particle density } \\ \omega & \text { angular velocity }\end{array}$

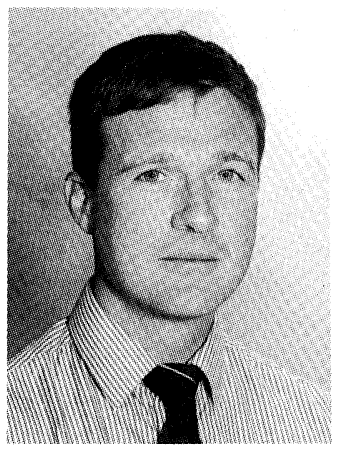

R.C. Bunge received his M.E. in Mining Engineering from the Technical University of Clausthal, Germany, and his Ph.D. degree in Mineral Engineering from the University of California at Berkeley, USA. He is now in charge of the process engineering laboratory of BMG Engineering AG, an environmental consulting firm in Zurich, Switzerland. Dr. Bunge is involved in the remediation of the contamination sites, in particular through soil washing technology.

Keywords: magnetogravimetric separation, magnetic fluid, magnetic buoyancy, gravity concentration 